\title{
Verification of Crime Due to Violence against Women in Karaj City and Effective Factors to Prevent It
}

\author{
Mahdi Momeni ${ }^{1}$ \\ ${ }^{1}$ Department of Criminal Law and Criminology, Payam Noor University, (PNU), Tehran, Iran \\ Correspondence: Mahdi Momeni, Department of Criminal Law and Criminology, Payam Noor University, (PNU), \\ Tehran, Iran. E-mail: drmomeni.law@gmail.com
}

Received: October 20, 2016

Accepted: December 21, 2016 Online Published: February 21, 2017

doi:10.5539/jpl.v10n2p30

URL: https://doi.org/10.5539/jpl.v10n2p30

\begin{abstract}
According to the new developments of criminology and approaches to crime victims. Victim - centered approach based on the conditions governing the development of crime and victim in order to prevent crime and reduce its implications are examined. A criminological finding suggests that some individuals for the reason that some of the special Features of biological and psychological and social victim are more at risk than others. Women often are in this context. The purpose of this research is to identify the types of violence in the city of Karaj.This research field of Karaj questionnaire about 384 women and using cluster and systematically implemented, at 2015-2016. The findings show that, there is violence in the mentioned society in various aspects. Most of the current violence is the psychological and sexual violence and economic violence is lowest. There is a direct and meaning full relationship between the notion authoritative man of his role and violence against women.Also there is an inverse and meaningful relationship between the contribution of the husband at home work and violence against women. There is a meaningful and direct relationship between men and women experience violence in their families and violence against women.
\end{abstract}

Keywords: prevention, violence, victim, women, city of Karaj

\section{Introduction}

Research and Evidence in recent years show that social pathologies have an increasing trend in communities. For its inhibition, we need to analyze and identify its causes and endeavor to resolve and decrease it in a scientific fashion and by its root.Discussion regarding victims of violence once used to be referred to in a diverting fashion. Yet, now a day one of the key discussions in criminology is attention to victims. This issue distorts the past triangular notion of crimes which focused on criminals and controlling them. Criminologists now believe that crime has more painful consequences that imagined. Victims were up to now forgotten in the process of punitive justice. Now a day, they play key role in this process. Currently, among the most modern findings in criminology shows that the criminal is no longer the main issue, but the victim is that is the person whose day to day activities may make him or her prone to crime (Yang \& Rush, cited in Salimi \& Davari, 2006, 245).

The importance of victim in the science of criminology manifests itself in cases such as occurrence of crime and crime rate at the society level. The reason is that some crimes are never recognized due to lack of complaint by victims and are not prosecuted. Now a day, victims and their needs have gained attention of criminologists at various national and international levels.Numerous researchers have presented definitions of violence. Golds and Strauss consider violence a behavior with explicit intention and purpose or a behavior that is attempted to be hidden. Yet, it is still perceived as inflicting physical pain to another person (Ezazi, 2001, 24). By this definition, psychological violence is not the topic of discussion. Yet, we know that violence is not just physical. In addition to accepting this definition, Martin also refers to psychological violence and speaks of open and hidden threats, constant surveillance of others, belittlement and the like (previous reference). Galtonick defines violence in a wider sense as any kind of behavior or structure that demeans an individual. In his opinion, we speak of violence when individuals are so much influenced that they assume their physical and psychological capabilities are less than its potential and truth (previous reference, p 26). In an announcement by the United Nations 1979 convention, there were clearly no laws in relation with violence and reference was only made to equal opportunity for working women and marital status and its dissolution. Yet, in 1993, the committee on women's rights supervised by the human rights committee ratified a decree by the United Nations in which violence 
against women was clearly banned. This announcement defines violence against women as follows: any kind of violent action against women by virtue of gender that leads to physical, sexual and psychological injury and harm and causes pain and abuse and threatens them and discriminates against them in society.

In the Beijing announcement (Peking), the Chinese World Convention Declaration, violence is defined as follows:Any kind of action against women that is gender related and its direct and indirect consequence is physical or psychological harm and abuse. These actions may be forceful and inflicted, by urge or discretion. They may occur both in public (society) or private (family life) form and will result in harnessing women's freedom.

This behavioral abnormality has a different cause and manifestations based on social, cultural and economic traits of societies and are witnessed in many countries irregardless of beliefs and opinions. Currently, in the most industrially advanced nations, women and children are in pain because of consequences of violence particularly in the family. Available statistics in this context show numerous tragedies in these countries.

In the country of Iran, because of cultural and religious characteristics, violence in streets, work place and the community against women is much less compared to the western world and the most common form of violence towards women are hidden. The reason is that many women refrain from expressing it due to reasons such as shame and protecting their image. What makes attention to this problem more serious is that in addition to violence against women being harmful on individual life, it also leads to negative consequences such as lack of security, social dysfunction and direct effect on rearing children. Additionally, this harmful phenomenon against women in society limits the possibility of appropriately taking advantage of their abilities and talents (Ghalibaf, 2009, 15-16).

People in power use violence and mal-intent against women and girls in private and public perimeters as a tool for stabilizing their power. In many cases, violence against women and girls are justified in the name of customs and culture. An internal network among social organizations attempts to under value the aggressive situation against women by relying on theories based on advantage of men and be-humbling of women. These ideas are also frequently taught and encouraged in schools. Legislative organizations also at times indirectly support the wrong doers and express lack of power to intervene. They consider the private perimeter of the family or close relationships out of their discretion. In some cases, the government also agrees with infliction of violence against women in private perimeters and considers this approach men's right for protecting order in the family. Family laws are frequently patriarchic and support men's dominance in the family (Mohammadi Asl, 2001, 15).

At times women for defending themselves or their children engage in physical violence against men. Yet, in most cases, women use verbal violence in reaction to men. Some women abuse and batter children; eventhough disciplining children is considered an important duty for women. Yet, aggressive behavior is beyond acceptable disciplining. In most cultures, infliction of physical or verbal harm by women is not acceptable. Most societies value manly aggressiveness in sports, politics and war. Yet, female aggressiveness is neither supported nor valued. Therefore, women are not able to stabilize their position by use of aggressive behavior.

Experts and specialists, well-fare social workers, legislators and sociologists believe that for protecting women in society and diminishing social pathologies, evaluation of causality and factors involved is necessary. In this way, opportunities will be secured. Factors under the influence of which individual rights are taken advantage of and victim is promoted and ultimately strategies for their revision are exposed. In this research, it has increasingly been endeavored to investigate causality, factors and conditions that lead to victim of women in the city of Karaj due to violence. Among the most important of these factors are education, occupation, age, differences, divorce, addiction and psychological disorder in spouse or parents.

\section{Definition of Violence}

In religious texts, violence arising from prejudice is considered the first behavioral abnormality in human relations and a cause for dismantling one member of the human race on earth (Maedeh Verse 27-31).Now a day, despite much cultural and social growth, the breadth and measure of violence is among significant human pains and concerns. One of the manifestations of violence in social organizations is directed towards women and children particularly the family unit. Violence against women in the history of humanity has been a global phenomenon. For this reason, in the last decade of the twentieth century, the United Nations has more actively engaged in omission of violence against women. In a scientific evaluation of violence against women, the first step is conceptual analysis and presentation of its operational definition. Presently, with evaluation of various definitions of it, the most comprehensive will be over told. Violence in the lexicon has been defined as anger, bad word of mouth, viciousness and being opposed to leniency and softness. Violence can be considered a harmful behavior performed by a person for advancement of his or her objectives and it does not just have a 
physical (bodily) form. Instead, it may have psychological dimensions (such as swearing, belittling, excluding individuals, yelling and scolding, sexual battery and abuse, rape) and economic dimensions (such as breaking home equipment) (Kar, 2000, 292). The term violence against women refers to any kind of aggressive behavior based on gender that leads to or may lead to occurrence of psychological and physical harm that is painful and abuse of women such as threats to the mentioned, forceful or discretionary seclusion in special situations and aborting freedom in public and private livelihood (Raisi Sartashnizi, 2005, 49). In 1991, the National Committee of Violence against Women in Australia has defined violence as follows: a behavior executed by men for controlling their victims' activities that leads to psychological, physical and sexual harm and social or economic seclusion such that the woman is placed in position of fear and horror (Arefi, 2003, 140).

\section{Methods of Inflicting Violence}

Generally with violence, we are witness to various verbal and behavioral patterns. Yet, we need to be aware that on the one hand, methods of violation of rights do not have similar influence on victims. Therefore, we can almost never say that what kind of violence is worse than the other and the degrees and classification of methods and forms of violence should not lead to lack of concern about other ones that are apparently mild in format (Nour Mohammadi, 2010, 37).

\subsection{Physical Violence (Body Injury)}

In this kind of violence, the appearance (corpus) and body of the victim is harmed. Considering the impact of physical violence, it can be considered the most explicit kind of violence that involves the victim, violator and researchers. Physical violence refers to any kind of aggressive behavior intended and purposefulleading to physical harm and injury. Its examples include: blows, battery, punching, biting, slapping, choking, blunt injuring using an object or the fist, knifing, gun shots wounds, mutilation, burying alive and homicide (Mohebi, 2001, 8).

\subsection{Psychological Violence (Emotional)}

Any kind of violent behavior and vocalization that harms the psyche, intellect and personality of an individual can be considered as psychological violence. It should be noted that this kind of violence can have more destructive effect compared to physical violence. Thus, it is natural that at times the victim is pushed towards suicide. Psychological violence can include a broad range of cases some of which are different based on mannerisms, traditions, cultures and beliefs of any society. This kind of violent behavior which is executed in the form of undue criticism, humiliation, belittlement, undignified verbalism, swearing and repeated threatening can lead to divorce or remarriage (Kar, 2000, 354).

\subsection{Financial Violation (Economic)}

In our religious culture, males are considered family supporters. One of their main responsibilities is provision of all necessary needs of family members. They are dutiful with regards to economic needs such as food, clothing, housing, education, health and other necessary needs.

If they do not uphold their duty or wish as a punishment instrument to refrain from provision of economic needs of their spouse and children, we encounter another form of violation namely economic in kind. Economic violation is a behavior that is intentional and purposeful and pressurizes the woman with affairs such as occupation, economy and assets and abuses and discriminates against her. Examples include: lack of payment of alimony, vileness, obsessive control of household expenses, forceful impingement on the woman's rights, lack of provision of necessary needs of the spouse and children and misuse or stealing of property and assets of the spouse (Mohebi, 2001, 8).

\subsection{Social Violation}

This case is a kind of violation in which women are excluded from social activities and interactions with others and is executed at a community level. This kind of violence places the woman in severe control of the man and socially excludes her such as prohibition of social relationships with family members, friends and limitations on education or occupation (Kar, 2001, 25).

\subsection{Sexual Assault}

One of the methods of executing violence is sexual abuse and assault. In some ways, it can be considered the worst type of violence. The reason is that it leaves numerous physical and psychological side effects. Sexual assault refers to any kind of anti-social behavior. It begins with touching and advances to the stage of rape. Women who are subjected to sexual assault suffer from psychological, neurological and emotional injury which influences their entire attitude towards the male sex (Kar, 2000, 346). 


\section{Social Learning Theory}

Evaluation of the topic of this research requires analysis of theories that have been introduced by legislators, psychologists and sociologists about the causality of violence against women.

\subsection{Social Learning Theory Revisited}

Albert Bandura emphasizes the trend of learning violence by way of observation and imitation. In this outlook, behavior is learned by imitation (imitation of behavior by family members and others) and also by way of direct experiences. If execution of this behavior in some environment leads to provision of a need or achievement of an objective, the probability of its repetition even without a reward will be high; in particular, if this behavior is set as an example and gives pride and value to the observer. The individual under the influence of his or her family gradually learns gender roles. Girls learn that the gender role of the adult goes along with acceptance of belittlement and dependence on men and boys learn that men should be better than women and follow their desires and wishes. The criticism of this theory was that if a behavior is based on learning, there should be a possibility to reduce or change it as well (Salimi \& Davari, 2006, 411).

\subsection{The Theory of Transaction}

Based on this theory, in the relationship among individuals, each tries to maximize his or her profit and minimize his or her expenditures. As a result, when for individuals involved in a relationship expenses and profits are at a balanced level the relationship endures. Gold describes violence and the theory of exchange as follows: in a malfunctioning relationship between a husband and wife the ratio of profit to expenses is in the following form:

A man batters his wife and in his opinion this is his right. The reason is that the woman is not behaving according to his wishes. The relationship between expenses (investments he has made for the family) and profits (behavior according to his wishes) is inappropriate. As a result, the husband attempts by way of violent behavior to create a balance in the family. Now, if with this strategy, he resolves the family dispute, considering the exchange theory, balance is achieved for him. The interaction of profit and loss for the male is as follows: due to violent behavior, the wife will refrain from disobedience and the man will reach his goals. Since he has become able to overlook the flow of life, his personal valuation and self esteem also increases (Motamedimehr, 2001, $35)$.

\subsection{Social Regulation Theory}

In the opinion of Hershey, delinquency occurs when the individual's commitment to society is weakened or totally obliterated (Meshkati \& Meshkati, 2002, 12). Social regulation theory emphasizes on crime and violence which occur by individuals whether it be intrinsically motivated or influenced by environmental factors. What is important in this view point is that individuals for reaching a goal or power, gravitate towards use of force. The second principle is that social regulation serves as a barrier against power seeking and violence. The reason is that humans gravitate towards crime, homicide and abnormal behavior without social impingements and society should create a regulatory mechanism for them. In this theory, delinquent behavior does not need external pressure, but lack of social regulation leads to its occurrence (Raisi, 2003, 141).

\subsection{Functionalist Approach}

The opinion of functionalists pertaining to the family explains violence. They consider the family as a group in which individuals with different genders and age groups interact with each other for a prolonged time. Unlike other groups, in this one, the hierarchy is not based on specialization, qualification and dignity. On the other hand, it is based on gender, age and economic power. Presence of different interests in this non homogeneous group leads to creation of open or hidden conflicts.

These conflicts destroy appropriate familial traits and functions. Thus, any action for resolution of family conflicts, eventhough at times with use of power and in some cases violence is justified and necessary (Ezazi, 2001, 69). Parsons, one of the founders of this theory, emphasizes gender roles and considers the father the family leader and the mother the internal family manager (Ezazi, 2001, 71).

\subsection{Cultural Theory}

The theory of culture of violence is among others that explain spouse abuse. This theory states that the basis of various kind of malicious behavior such as spouse abuse, child abuse and geriatrics abuse forms by society. In this view point, culture is a social legacy of the past that influences present and future behavior. Dubash and Dubash have found clear correlation between aggressive behavior by men towards women and social norms. Men who maltreat their wife live in a culture in which the governance of men is much more than women. Among other characteristics of this culture is influence of men's violence, chauvinistic dominance and obedience by 
women. Therefore, in patriarchic societies, use of power for stabilizing chauvinistic dominance is legitimate. In some communities, when social order is endangered, to re-establish it violence is used. This type of social regulation, namely execution of violence, for survival of social order occurs both at the macro and individual (familial) level (Ezazi, 2001, 71).

\subsection{Social Construction Theory (Tension and Conflict)}

The more tension inducing events and situations that threaten the family are, the higher will be the probability of occurrence of aggressive behavior in the family. The latter has been confirmed in research about the relationship between tension and violence. The reason is that families that are less at risk of various kinds of tensions show less violence. In these investigations, tension manifests in the form of events at the work place, breaking the law and summons to the prosecution office and court, illness and other family malfunctions. The reason is that the probability of occurrence of such behavior is increased when the aggressor by way of observation or experience reaches the conclusion that execution of violence is a correct response at times of conflict. In addition, he should believe in legitimacy of such behavior and assume that using violenceenhances him more positively (Moazemi, 2007, 82).

\subsection{Feminist Approach}

The main idea in this theory is that direct and indirect social processes act in support of a kind of specific social order and patriarchic family structure. Patriarchy leads to placement of women in lower positions and historical formation of systemic aggressive models against women (Gold \& Strauss, 2000, 128). In this respect, the main reason for marital violence lies in traditional familial structure which is a kind of power seeking chauvinistic order. In addition, feminist researchers consider male superiority explicitly the main reason for household violence. Feministic analysis of battery of women by men is in many ways a criticism of patriarchy (Strauss \& Udanis, 1990, $841 \&$ 825). Dubash and Dubash in a historic investigation have followed the phenomenon of female battery by males from the time of enlightenment in Europe up to today and have shown that women in patriarchic systems have constantly been dominated by men by use of physical violence and remain so up to present (Ezazi, 2001, 77).

\subsection{Resources Theory}

Resources theory introduced in 1971 by William Goode emphasizes the matter that frequently men have the economic resources in their hand and this issue leads to their advantage in familial relationships. While women are mainly dependent on their spouses when it comes to economic resources and also have the responsibility of taking care of the children. If they leave men, they will encounter economic difficulty. Thus, they remain in conflicting relationships. Women's dependence means access to limited resources for them and less power on men. If one of the couple seeks power, he or she generally selects abusing and abuse has various kinds such as: threats, fear, exclusion, child abuse and financial limitations. Yet, couples equal in power and resources experience less conflict and if so resolve it with non aggressive methods (Molaverdi, 2006, 74).

\subsection{Gender Identity Theory}

Mitchell Fuko believes that in Western societies individuals identify themselves based on their gender and consider them sexual beings. They extend this concept of self-genderism to their entire ethical and traditional livelihood. This means that each individual for understanding how to behave and what expectations of society is from him or her, initially looks for a model by which a person with his or her gender should behave in accord with (Tavakoli, 2003, 80). In the opinion of Michael Camille also chauvinism is a collection of concepts formed in the process of our relationship with ourselves, others and our universe. Chauvinism has a social source and does not arise from our biological structure. On the other hand, it is a product of culture (the same). Camille believes that sexual violence takes source from three factors: 1) Irrelevant and unjust social power of men and in some cases, their use of violence (against women and also other men, themselves and nature ...) to protect this power or at least promote their sense of it; 2) Sense of entitlement to power and advantage which is experienced by most men and 3) Social, legal and culturalpermission to be sexually violent which more or less exists in most patriarchic societies (the same, 83).

\subsection{Environmental Theory}

Transfer of the environmental concept to familial violence is because of interest in presenting a union between psychological and sociological theories. In this theory, social acceptance of children is explained at three different levels: unmediated environment, social networks and globalistic system (ideologic). Family as the living environment has especial role in children's learning and their relationship with the external world (neighbors and local) is the index of fruition of socialization (Nazari Jeirani, 1999, 134). If the family is unable 
to make relations with the external environment, violence (particularly against children) may arise. Thus, when mutual relationship does not exist between parents-child, family and the environment, violence arises. At the same time, it is possible that lack of preparation of guardians for accepting their role, their excessive expectations from children and their inabilities in encountering environmental difficulties also can lead to violence. Yet, this condition by itself is not sufficient for arising of conflict. In the environmental theory, what is important in creation of conflict is that social interpretations make this act acceptable appearing and the family's social network as well is determinant in occurrence of conflict. The reason is that parents admonish their children only when a normative system encourages them to do so or social support organizations do not guide them in the context of developmental problems of children and does not remove some load from the shoulders of parents.

\section{Hypotheses}

- Direct correlation exists between male imagination of its role as the power seeker and female imagination of its role as subordinate and violence against women.

- Violence against women in Karaj is caused by various social, psychological or economic and cultural factors.

- Creation of job opportunities, social well-fare and promotion of overall culture as fundamental strategies in the matter of preventing violence against women.

\section{Statistical Population}

The statistical population of this research is all women in the city of Karaj in the 18-67years of age range. Generally, this group is more active compared to other age groups in city space and the probability of their victim is higher. With attention to the Cochrane formula with error of $d=0 / 05$, the sample size of this research was estimated at 385 individuals. After gaining confidence in validity and reliability of the measurement instrument of this research and final preparation, questionnaires were randomly distributed among the participants in public locations and door to door at their homes. Research data was collected using questionnaire. To determine instrument validity, external validity was examined and it was evaluated by experts and with their input, deficiencies were resolved. Additionally, the technique of well known groups was used for determination of content validity of the questions and items. Also, for determination of reliability the Cronbach's alpha coefficient was used which was calculated at $0 / 888$ for this research.

\section{Sampling Method}

\subsection{Sample Size Determination Formula}

For determination of sample size the following two stages were followed:

The overall sample size formula (Cochrane formula) is as below

$$
n_{0}=\frac{Z_{\alpha / 2}^{2} \operatorname{var}(\theta)}{d^{2}}
$$

Where in the above formula the normal standard value for $Z_{\alpha / 2}$

is equal to 1.96 with a confidence percentage of $100(1-\alpha)$

The variance of the estimated parameter is $\operatorname{var}(\theta)$

And the level of error is $\mathrm{d}$.

If the sample size is unlimited, the above formula is used; but, if it is specific and equal to $\mathrm{N}$, the above formula is adjusted as follows:

$$
n=\frac{n_{0}}{1+\frac{n_{0}}{N}}
$$

Off course, the value for $\operatorname{var}(\theta)$ is regularly unknown. Yet, if the research population (one of the parameters of

the research) has two forms, we can consider $\operatorname{var}(\theta)=p q$. The advantage of this action is that we can 
consider the maximum value for $\operatorname{var}(\theta)$ (the maximum value possible) which occurs when: $p=q=\frac{1}{2}$.

$$
\begin{gathered}
n_{0}=\frac{Z_{\alpha / 2}^{2} \operatorname{var}(\theta)}{d^{2}} \\
n_{0}=\frac{(1.96)^{2}(0.5)(0.5)}{(0.05)^{2}}=384.16
\end{gathered}
$$

Therefore, with an error level of $\mathrm{d}=0 / 05$, sample size needed in this research was estimated to be 385 individuals. In this sample of 385 individuals, 23/6 percent were between 18-25 years old, 26/5 percent between 26-33, 20/8 percent between 34-41, 16/6 percent between $42-49$ and 12/5 percent were between 50 to 67 years of age. Also, 43/9 percent were single and 56/1 percent married. Education level in 34/5 percent was diploma and Associate's of Arts, 53 percent Bachelor's degree and12/5 percent Master's degree. Age of marriage in 44/4 percent was 18-20 years of age, in 48/1 percent 21-23 and in 7/4 percent higher than 24 years of age.

\section{Method of Data Analysis}

Data analysis was a multi stage process in which information gathered using research instrument from the statistical sample was summarized, codified, classified and ultimately processed to be able to analyze them and find correlations between them for testing the hypotheses. In this process, the data was refined both conceptually and from an experimental point of view and various statistical methods had significant role in interpretation and extrapolation of information. For data analysis, initially descriptive statistics was used where demographic variables of the research including education level, age and ... were processed. Next, according to hypotheses under consideration, to evaluate their accuracy relevant statistical tests were used with the help of SPSS software.

\section{Definition of Concepts and Indices}

\subsection{Independent Variables}

Independent variables were initially introduced for evaluation and next prepared in the frame of questions and items in a four choice format using the Likert scale and distributed among respondents. Independent variables including male imagination of self as power seeking, female evaluation of her role as subordinate, upholding gender roles, socio-economic status and male and female experience of violence in the family.

Male assumption of role of self being power seeking: All actions that are signs of maleness and his traditional role which in this research includes power, decision making regarding income and expenditures, being more intellectual compared to women, lack of agreement with women's continuing education and performing manly deeds.

Female assumption about her own role: Includes all actions that are signs of womanhood and her traditional role. The assumption defined by society in this research includes qualities such as being obedient and subordinate, emotional and sensual, high endurance and patience and performing all activities relevant to the gender role of a woman such as housekeeping and child rearing.

Upholding gender roles:Gender (female, male) has a social and cultural construct that is acquired actively in the process of socialization. Therefore, gender goes beyond intrinsic, biologic and/or psychiatric traits. In patriarchicfamilies, gender is based on sexuality such that boys have higher male and girls higher female identity (Mambani \& Hosseinzadeh, 2011, 69).

Socio-Economic Status: To evaluate socio-economic status of participating women, three indices of education, income and occupation were used and after standardization of the three mentioned topics and combining them the socio-economic status variable was obtained.

Experience of Violence by Women and Men:In the process of socialization in the family, individuals place parents as models and watch violence in the family and learn it. Such aggressive behaviors may arise from the side of the father and mother interacting with each other or the person may him or herself be subjected to violence by one of them. In this research, this experience includes observation of the dispute between parents, battery or belittlement by parents or spouse, battery by brother or sister or watching mother being beaten up. 


\subsection{Dependent Variable}

In this research, the dependent variable is violence which includes 5 dimensions: physical, psychological, sexual, social and economic.

\section{Research Results}

Topics presented in this section are results of analysis of interviews with a number of women from Karaj. It has been endeavored to express topics designed regarding victim of women and factors influential on it in their own language. Conditions and situations women face in encountering society and interaction with its members may be a different experience lived in their social life time. Women's interpretation of day to day living, how they adapt with it and threats and dangers of social life, all may be unique for each individual or have much commonalities and similarities produced by living in a similar society or in other cultural, political, economic and social situations and conditions. The age range of the respondents was between 18 and 67 where a higher percentage was between the ages of 26 and 33 and 43/9 percent were single and 56/1 percent were married. Marital age in 44/4 percent of the participants was between 18 and 20, in 48/1 percent 21 to 23 and in 7/4 percent higher than 24 years of age. It can be concluded that a higher percentage of respondents had a marital age between 21 to 23 years old. Education level in 34/5 percent of the respondents was diploma and Associate's of Arts, in 53 percent Bachelor's and in 12/5 percent Master's degrees. We conclude that a higher percentage of the participants were educated up to Bachelor's degree. In total, the results of analysis of interviews show that they consider factors most important in victim of women such as lack of carefulness, kind of daily activities, kind of behavior and dress code, violence in the private and public domain, familial dysfunction and weakness in socialization, dysfunctional social support laws, lack of learning self defense techniques, unorganized city environment, existence of high crime areas that are hidden such as under passages and dark walk ways, economic failure such as poverty and unemployment alongside biological and physiological traits. In this research, 5 dimensions of violence against women were evaluated which include physical violence, psychological violence, sexual abuse, social disdain and economic violation. Evaluation of these 5 dimensions of violence is shown in the following table:

Table 1. Description of various kinds of violence against women

\begin{tabular}{llll}
\hline Kinds of violence & Experience of violence & Number & Percentage \\
\hline \multirow{4}{*}{ Physical violence } & None-little & 159 & 41.3 \\
& moderate & 209 & 54.3 \\
& Much-Very much & 17 & 4.4 \\
Psychological violence & None-little & 131 & 43.3 \\
& moderate & 235 & 61.4 \\
& Much-Very much & 19 & 4.9 \\
Sexual abuse & None-little & 133 & 34.5 \\
& moderate & 178 & 46.3 \\
& Much-Very much & 74 & 19.2 \\
Social violence & None-little & 140 & 37.4 \\
& moderate & 144 & 38.5 \\
& Much-Very much & 90 & 24.1 \\
Economic violation & None-little & 165 & 42.9 \\
& moderate & 207 & 53.7 \\
& Much-Very much & 159 & 41.3 \\
& None-little & 209 & 54.3 \\
Total & moderate & 17 & 4.4 \\
& Much-Very much & 131 & 43.3 \\
\hline
\end{tabular}

Physical Violence: Considering the results obtained, 41/3 percent (159 individuals) of women respondents 
reported experiencing little physical violence, 54/3 percent (209 individuals) moderate and 4/4 percent (17 individuals) high amount. Overall, it can be concluded that a major portion of women experienced moderate level of physical violence. Also, 58/7 of the women had experienced physical abuse.

Sexual Abuse: The results showed that $34 / 5$ percent (133 individuals) of participants experienced little sexual abuse, 46/3 percent (178 individuals) moderate and 19/2 percent (74 individuals) high levels. In total, major portion of women participants had experienced moderate levels of sexual abuse and 65/45 percent of women had experienced sexual violation.

Social Violation: The results show that $37 / 4$ percent (140 persons) of women participants have undergone little social violation, $38 / 5$ percent (144 persons) moderate and 24/1percent (90 persons) high levels. In total, it is shown that a major portion of women have tolerated moderate levels of social violation and $62 / 5$ percent have experienced it in total.

Economic Violation: Considering the results obtained, it is noted that $42 / 9$ percent (165 individuals) of women participants have experienced little economic violation, 53/7 percent (207 individuals) moderate and 3/4 percent (13 people) have experienced high levels of economic violation. In total, it is noted that a major portion of women have experienced moderate levels of economic violation and 57/14 percent economic violation in general.

Overall, 61/96 percent of women in the city of Karaj have witnessed violation.

\section{Discussion and Conclusion}

Violence and crime as a social phenomenon and issue has gained attention of experts in various fields. Each has evaluated this issue from various aspects which has led to numerous classifications. In this research, emphasis has been placed on various kinds of violence against women. Results of the research show that all dimensions of violence against women more or less existed in the research sample. The most common form of violence in the studied population is related to psychological form and sexual abuse which constituted 65/97 and 65/45 percent and the least form was economic with 57/14 percent of the participants involved. Since sexual issues are considered private matter in traditional societies such as the sample in this study, women have no interest in retelling it. It can be stated that sexual abuse is at moderate level. If privacy considerations are at mind, it can be stated that sexual abuse exists at a high level. Results show that the level and severity of violence in the city of Karaj is at a moderate level. This statistics shows that one third of the respondents have undergone some kind of violation. Since violence in the family is a private and hidden matter and individuals do not like to talk about it, it can be stated that little levels of violence may really represent moderate amounts and moderate levels high amounts. Thus, violation in Karaj mayexist at least at a moderate level.

Based on the theory of social acceptability of gender, the process of socialization in families internalizes female and male gravitation where generally woman are portrayed as calm, obedient, emotional and men independent, able and perseverant. Results of this research show that the higher the female assumption that she has a subordinate role, the overall level of violence decreases. This matter refers to acceptance of violence by women. In other words, the more they neglect it, the level of violence against them decreases. Additionally, the theory of patriarchy worships chauvinistic identity which is aggressiveness and respects those who execute chauvinistic roles, gives them power and advantage and internalizes the issue that ordering and if required, degrees of violence by men is natural and due. Women are expected to be subordinate. Chauvinistic power and precise overlooking on all affairs such as women's thoroughfare and their behavior leads to protection of family cohesiveness and acknowledgement of society's values and regulations. Therefore, based on this theory, results of this research show that the reason for decreased violence in the population studied, where obedience is assumed to be high, is also its acceptance. Additionally, based on this theory, another hypothesis in this research shows male imagination of his role as powerful and its relation with violence is confirmed. The reason is that the findings show there is direct and meaningful correlation between this type of thinking and various dimensions of violence such as psychological, physical, social, economic and overall violation. In other words, increased chauvinistic approach by men leads to increased violence in the family which agrees with research by Dehghanfard and Raisi. The second hypothesis shows inverse correlation between socio-economic status of men and violence against women. Based on the results obtained from testing the hypotheses, inverse correlation exists between socio-economic status of men and husband violence against women. This result confirms the theories of Goode. He believed that physical power and threatening to use it is similar to having money and personal outlooks that individuals take advantage of for controlling other people's actions. In fact, people rely on physical power when they lack any other resources or those required for success (Yantes \& Carreira, 2006, 367). Results of evaluating the variable of socio-economic status show that men who in terms of occupation, income and 
education are at lower levels and belong to weak socio-economic strata execute more violence towards women. When the individual wishes to exert power over others, yet his or her resources (such as education, occupation, income and skills) are at a low level, he or she uses violence for stabilizing his or her status. Low income pressurizes individuals and men who lack any other source for maintaining their dominance over the family, for its persistence rely on any action and ultimately violence against women. Previous research by Erchuck and Rosenfeld (1994) and Oddinkra (2007) also support this hypothesis.

Test of the third hypothesis confirms an inverse correlation between social support resources accessible to women and male violence against them. Theory of resources states that the family unit or relations can change the risk of violence against women particularly when a broad kinship system or strong inter- generational relationship exists. Social relations between women and family and friends can strengthen her connections with kinships and encourage discussion about male-female differences. Results of evaluating the variable of social support resources accessible to women show that in this research, social resources have inverse and negative influence on violence against women. The latter means that the higher the level of closeness in social relations between women, family and friends, the lower the level of violence against them will be. The reason is that women with close family and friends have shelters to refuge to at times of crisis or violence against them. Thus, women with no relationships with their friends and family remain in complex relationships. Finally, based on the theory of dominant learning, human behavior is learned by way of observation and by virtue of the process of modeling. In this view point, the social learning phenomenon is mainly a consequence of experiences gained by watching behaviors of another individual and its side effects. In the opinion of Bandura, children learn violence from their parents. Based on the theory of transfer between generations which is based on social learning theory, individuals who experience violence in their family in childhood or witness it extensively in adulthood will utilize it. Results of this research also show that direct and meaningful correlation exists between violence against women in the prime family and psychological, physical, sexual, social, economic and overall violations. Additionally, results of research by Nazparvar Begrezaii, Emamipour, Dehghanfar, Golds and John Hubers show that meaningful correlation exists between experiencing and witnessing violence in the prime family with level of violence against women. Violation is an ethical issue with roots in rearing and at times mutual human relations. Therefore, its resolution depends on revision of outlooks, habits, emotions and behaviors of the two genders. Thus, thinking and outlook towards women should be changed accordingly. According to the United Nations Convention, such measures should be adopted for combat against the concern of violation against women that the entire society becomes free of such. Acquisition of such measures in the first place is in the hands of every country's legislation, well-fare organization, social insurance organizations, civil sectors and public organizations for women and their well-being. Below, suggestions in line with the above goal will be presented:

- Guarantee of legal and judiciary support and security and police organizations for victimized women.

- Formation of educational committees for women and men with attendance of specialists and counselors in family affairs to increase gender knowledge and promote level of addressing of and reaching women and men. This committee can constitute of volunteers and their main duty is design of open discussions revolving around reasons for violence and how to limit this kind of attitude in familial relations and between men and women.

- Allocation of public budget for training expert cadres in the family and social counseling affair.

- Formation of safe homes for women and emergency phone centers, voluntary teams of women and men national guards for support of violated girls and women.

- Creation of committees formed of men for intellectual and psychological counselingof men who commit violence. To encounter psyche of aggressiveness combined with violence in men. Such organization should be a place for transfer of male experience and education. Presence of specialists in such organ is a definite recommendation.

\section{References}

Arefi, M. (2003). Descriptive evaluation of home battery against women in the city of Urmieh. Women's Studies, $1(2)$.

Ezazi, S. (2001). Family violence (battered women). Tehran: Sali Publications.

GellS, R. J., \& Straus, M. A. (1979). Detemporary of Violence in the family. In W. R. Burre, R. Hill, F. J. Nye, \& L. I. Reiss (Eds.), contemporary theories a bout the family. New York: Free Press.

Ghalibaf, A. (2009). Evaluation of perception of women of their experiences relative to various kinds of home abuse. MS Dissertation, University of Tehran, Tehran. 
Kar, M. (2000). A research with regards to violence against women in Iran. Tehran: Roshangaran (Women's Studies) Publications.

Meshkati, M., \& Meshkati, Z. (2002). Evaluation of influence of internal and external familial factors on delinquency in adolescents. Journal of Society of Sociology, 4(2), 14-21.

Moazemi, S. (2001). Report of violence against women project in the counties of Sistan and Balouchestan-Kurdistan-Golestan-Boushehr and the county of Islamshahr. College of Law and Political Sciences, University of Tehran.

Mohammadiasl, A. (2001). Management of dysfunction amongst Iranians and its social roots. Farhange Tosee Journal, 10(50).

Mohebi, F. (2001). Social pathology for women (violence against women). Journal of Women's Book, 5(145).

Molavrdi, S. (2006). Anatomy of violence against women. Tehran: Hoghoughdan and Daneshnegar Publications.

Motamedimehr, M. (2003). Support of women against violence (English Criminal Policy). Tehran: Barge Zeitoun Publications.

Nazari Jeirani, M. (1999). Spouse abuse, a social dilemma for women. Collection of Women's Articles on New Research Evidence, University of Alzahra.

Nour Mohammadi, Gh. (2010). Abolition of violence against women in the view of science and religion. Tehran: Riasat Jomhouri (Center for Women and Family) Publications.

Raisi, J. (2003). Youth and behavioral abnormalities. Journal of Social Sciences, (21), 138-158.

Raisi, T. E. (2002). Violence against women and factors influencing it (Case study: county of Shahrekord). Journal of Women's Studies, 66(3), 1-45.

Salimi, A., \& Davari, M. (2006). Sociology of deviation. Collection of Studies on Deviation and Social Control, Hozeh and University Research Center, Ghom.

Straus, M. A., \& Yodanis, C. L. (1990). corporal punishments in adolescence and physical assaults on spouses in later life: what accounts for the link? Journal of marriage and the Family, 58, 825-841. https://doi.org/10.2307/353973

Tavakoli, N. (2003). Sexual identity of spouses and violence against women in the family. PhD Dissertation, Azad Islamic University, Tehran.

Yount, K., \& Carrer, J. (2006). Domestic violence against Married women in combodia. Social Forces, 25(1), 354-387.

\section{Copyrights}

Copyright for this article is retained by the author(s), with first publication rights granted to the journal.

This is an open-access article distributed under the terms and conditions of the Creative Commons Attribution license (http://creativecommons.org/licenses/by/4.0/). 